\title{
Chemical properties of long gamma-ray bursts progenitors in cosmological simulations
}

Pellizza L. J. ${ }^{a b}$, Artale M. C. ${ }^{* a b}$ and Tissera P. B. ${ }^{a b}$

a Instituto de Astronomía y Física del Espacio, Buenos Aires, Argentina

${ }^{b}$ Consejo Nacional de Investigaciones Cientícas y Técnicas, Argentina

${ }^{c}$ Max Planck Institute for Astrophysics visitor

E-mail: (pellizza, mcartale, patricia) @iafe.uba.ar

\begin{abstract}
In this work, we investigate the chemical dependence of the progenitors of long gamma-ray bursts. Using hydrodynamical cosmological simulations consistent with the concordance $\Lambda$-CDM model which include star formation, chemical enrichment and supernova feedback in a self-consistent way, and assuming that these bursts are produced by a subset of massive stars (possibly with distinct chemical properties), we compute the LGRB rate at different redshifts. Introducing prescriptions for their peak isotropic luminosity function and intrinsic spectrum, and using a Monte Carlo scheme to model their detectability by different high-energy observatories, we compute the distributions of the burst observables (peak flux, spectral peak energy) and compare them to actual data. Our preliminary results show that a possible chemical dependence for LGRBs progenitors cannot be ruled out, but it might be more complex than the usually assumed metallicity cut-off.
\end{abstract}

Gamma-Ray Bursts 2012 Conference-GRB2012,

May 07-11, 2012

Munich, Germany

\footnotetext{
* Speaker.
} 


\section{Introduction}

The nature of the progenitors of long gamma-ray bursts (LGRBs) and the LGRB-star formation connection has been investigated both observationally and theoretically for the last decade (e.g. [14] and references therein). Several studies have been devoted to investigate LGRBs as possible star formation tracers obtaining dissimilar results, which still makes the topic a matter of discussion. Although it is clear now that LGRBs are generated by massive stars, and consequently can be associated to star forming regions, the dependence of LGRB production on the chemical abundances of the progenitors is still controversial. Some authors propose that the chemical-dependence hypothesis would allow to explain both the properties of the hosts, and the LGRB redshift and peak flux distributions $[4,10,7,3,1]$, while others claim that an LGRB rate that follows star formation (i.e., with no dependence on the abundances of the progenitors) does the same job [5,9]. There are several reasons behind this disagreement, among them the poorly constrained star formation rate (SFR) at high redshift, its chemical dependence, the amount of dust obscuration in LGRB hosts, and the lack of a large sample of LGRBs confirmed to be at high redshift. One approach to the problem is to assume a co-moving LGRB rate proportional to the co-moving SFR, eventually with a redshift or metallicity-dependent proportionality factor, compute a simulated LGRB population (redshifts, peak luminosities, intrinsic spectral parameters), and compare the predictions of the model to gamma-ray observables such as the distributions of peak fluxes, redshifts and observed spectral parameters $[4,8,10]$. In this approach, the co-moving SFR and its metallicity dependence are usually obtained from analytical models [6]. In this work, we apply the aforementioned method to the SFR provided by hydrodynamical cosmological simulations of galaxy formation and evolution consistent with the concordance $\Lambda$-CDM model. These simulations include star formation, chemical enrichment and supernova feedback in a self-consistent way, hence they provide a consistent description of the evolution of the SFR and the chemical abundances of the newborn stars.

\section{Cosmological Simulations}

In this work, we use three hydrodynamical simulations consistent with the concordance $\Lambda$ CDM model, computed using a version of GADGET-3 that includes star formation, metal-dependent cooling, chemical enrichment, multiphase treatment for gas particles and supernova feedback (SNII, SNIa) $[11,12]$. The simulated volume is a periodic box of $10 \mathrm{Mpc} h^{-1}$ comoving side, where $h$ is the Hubble parameter. The mass inside this volume is described initially by with $230^{3}$ particles of dark matter with a mass of $5.93 \times 10^{6} M_{\odot}$, and $230^{3}$ particles representing the gas, with initial masses of $9.12 \times 10^{5} M_{\odot}$. Initial gas abundances are $X_{\mathrm{H}}=0.76, X_{\mathrm{He}}=0.24$, and the code follows the evolution of the mass fractions of ${ }^{1} \mathrm{H},{ }^{2} \mathrm{He},{ }^{12} \mathrm{C},{ }^{16} \mathrm{O},{ }^{24} \mathrm{Mg},{ }^{28} \mathrm{Si},{ }^{56} \mathrm{Fe},{ }^{14} \mathrm{~N},{ }^{20} \mathrm{Ne},{ }^{32} \mathrm{~S},{ }^{40} \mathrm{Ca} \mathrm{y}$ ${ }^{62} \mathrm{Zn}$.

We used three simulations (A, B and C) with different star formation efficiency and supernova feedback parameters, which results in different star formation and chemical enrichment histories for the interstellar medium (ISM). In Table 1 we present, for each simulation, the critical density $\rho_{\mathrm{c}}$ over which star formation is allowed, the energy feedback to the ISM per supernova $\varepsilon$, and the 
Table 1: Properties of cosmological simulations.

\begin{tabular}{llrr}
\hline Simulation & $\rho_{\mathrm{c}}\left(\mathrm{g} \mathrm{cm}^{-3}\right)$ & $\varepsilon\left(\times 10^{51} \mathrm{erg}\right)$ & $f_{\mathrm{m}}(\%)$ \\
\hline A & 0.032 & 0.7 & 50 \\
B & 0.032 & 0.4 & 80 \\
C & 0.32 & 0.7 & 70 \\
\hline
\end{tabular}

mass fraction of metals that goes to the cold phase of the ISM in a supernova explosion $f_{\mathrm{m}}$. The cosmological parameters are $\Omega_{\Lambda}=0.7, \Omega_{\mathrm{m}}=0.3, \Omega_{\mathrm{b}}=0.04, \sigma_{8}=0.9$ and $h=0.7$.

\section{Synthetic LGRB populations}

We aim at constructing synthetic populations of LGRBs under different assumptions about their progenitors, to compare their predictions about the distributions of LGRB observables with actual data. Assuming that LGRB progenitors are massive stars with metallicities $Z$ lower than a certain cut-off $Z_{\mathrm{c}}$, the number of LGRBs observed by a detector monitoring a solid angle $\Delta \Omega$ in the sky during a time $\Delta t$ is given by

$$
N_{\mathrm{obs}}=\int_{0}^{\infty} \frac{\Delta \Omega}{4 \pi} k \frac{\rho_{\mathrm{SFR}}\left(z \mid Z<Z_{\mathrm{c}}\right)}{1+z} \frac{d V}{d z} p_{\text {jet }} f_{\mathrm{obs}} d z
$$

where $\rho_{\mathrm{SFR}}\left(z \mid Z<Z_{\mathrm{c}}\right)$ is the co-moving SFR density at $Z<Z_{\mathrm{c}}$ at redshift $z, k$ is the mean number of LGRB progenitors per unit of newborn stellar mass, $d V / d z$ is the redshift derivative of the comoving volume at fixed solid angle, $p_{\text {jet }}$ is the probability that the LGRB jet points towards the observer (assumed to be constant), and $f_{\text {obs }}$ is the fraction of properly oriented bursts detected by a real instrument. As the detectability of a burst depends mainly on its peak flux, the value of $f_{\text {obs }}$ depends both on redshift and on the LGRB luminosity function and spectral parameters distributions. The metallicity cut-off $Z_{\mathrm{c}}$ is a free parameter of our model.

The integrand of Eqn. 3.1 divided by $f_{\mathrm{obs}}$ is the redshift distribution of LGRBs observed by an ideal detector on Earth. Hence, we used the data on the ages and metallicities of the simulated stellar populations to compute $\rho_{\mathrm{SFR}}\left(z \mid Z<Z_{\mathrm{c}}\right)$, and a Monte Carlo (MC) scheme to create an ideally observable sample of $10^{6}$ LGRB redshifts. Assuming a power-law isotropic luminosity function for LGRBs with slope $\gamma<0$, a Band [2] rest-frame LGRB spectrum with low and high-energy slopes $\alpha=-1$ and $\beta=-2.3$, and a lognormal spectral peak energy distribution with mean $\mu$ and dispersion $\sigma(\gamma, \mu$, and $\sigma$ are free parameters), we used a second MC scheme to assign isotropic luminosities $L_{\text {iso }}$ and spectral peak energies $\left.E_{\text {peak }}\right)$ to the sample. Each LGRB is now defined by the values of $\left(z, L_{\text {iso }}, E_{\text {peak }}\right)$, from which we compute the peak flux $P$ in BATSE and Swift energy bands and apply a third MC scheme to reject LGRBs according to the detector sensitivity at $P$ (taken from $[13,4]$ ). This effectively simulates the factor $f_{\text {obs }}$, and produces two samples (one for BATSE and the other for Swift) comparable to the observed ones.

\section{Preliminary Results}

For each simulation (A, B , C) we ran the aforementioned scheme producing a LGRB sample for several values of $Z_{\mathrm{c}}$ between 1 (no metallicity dependence) to $0.0002\left(0.01 Z_{\odot}\right)$. For each value 


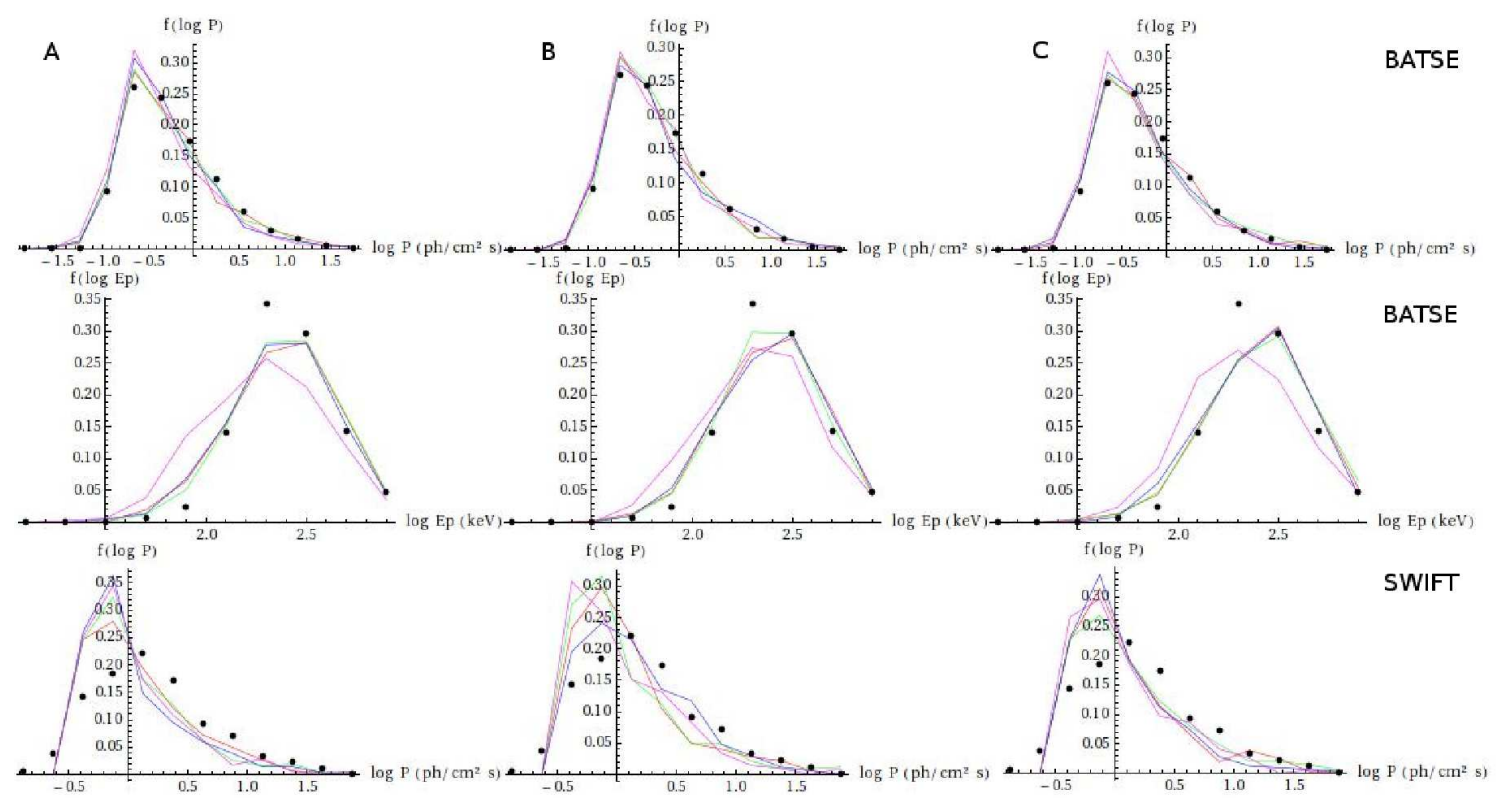

Figure 1: Preliminary results. Upper, middle and lower panels present the BATSE peak flux distribution, BATSE spectral peak energy distribution and Swift peak flux distribution, respectively. Each column shows the result of a different simulation (A, B, C from left to right). Black dots represent the actual data, while red, green, blue and magenta lines represents the outcome of our models with $Z_{\mathrm{c}}=1,0.01,0.005$ and 0.0002 , respectively.

of $Z_{\mathrm{c}}$ we fitted the other free parameters to reproduce the BATSE peak flux and spectral peak energy distributions.

Our results (Fig. 1) can be described with best-fit parameters $\gamma=-1.6, \mu=2.85$, and $\sigma=0.2$, in agreement with those of [4]. We observe that the peak flux distribution in BATSE is described by any model, regardless of the metallicity of the progenitors. However, Swift observations are not well fit by any model, which always show an overabundance of low-flux LGRBs. Our preliminary results can neither confirm nor rule out a metallicity dependence of the LGRB progenitors, if the latter is described by a simple cut-off. However, the discrepancy of Swift data with every model suggests that a more complex metallicity dependence might be necessary to give an answer to these questions.

\section{References}

[1] M. C. Artale, L. J. Pellizza \& P. B. Tissera, Chemical abundances and spatial distribution of long gamma-ray bursts MNRAS 2011 (415) 3417

[2] D. Band, J. Matteson, L. Ford et al., BATSE observations of gamma-ray burst spectra. I - Spectral diversity ApJ 1993 (413) 281

[3] N. E. Chisari, P. B. Tissera \& L. J. Pellizza Host galaxies of long gamma-ray bursts in the Millennium Simulation MNRAS 2011 (408) 647

[4] F. Daigné, E. M. Rossi, R. Mochkovitch, The redshift distribution of Swift gamma-ray bursts: evidence for evolution MNRAS 2006 (372) 1034 
[5] J. Elliott, J. Greiner, S. Khochfar, P. Schady, J. L. Johnson \& A. Rau, The long $\gamma$-ray burst rate and the correlation with host galaxy properties A\&A 2012 (539) 113

[6] A. M. Hopkins \& J. F. Beacom, On the Normalization of the Cosmic Star Formation History ApJ 2006 (651) 142

[7] S. E. Nuza, P. B. Tissera, L. J. Pellizza, et al. The host galaxies of long-duration gamma-ray bursts in a cosmological hierarchical scenario MNRAS 2007 (375) 665

[8] L. Pellizza, S. Nuza, P. Tissera, et al., Proceedings of the First La Plata International School: “Compact Objects and their Emission”, eds. G.E. Romero \& I. Andruchow, 2008109.

[9] C. Porciani \& P. Madau On the Association of Gamma-Ray Bursts with Massive Stars: Implications for Number Counts and Lensing Statistics ApJ 2001 (548) 522 [astro-ph/ 0008294 ]

[10] R. Salvaterra \& G. Chincarini, The Gamma Ray Burst Luminosity Function in the Light of the Swift 2-year Data ApJ 2007 (656) 49 [astro-ph / 0612278 ]

[11] C. Scannapieco, P. B. Tissera, S. White \& V. Springel, Feedback and metal enrichment in cosmological smoothed particle hydrodynamics simulations - I. A model for chemical enrichment MNRAS 2005 (364) 552 [astro-ph/ 050544 0v5]

[12] C. Scannapieco, P. B. Tissera, S. White \& V. Springel, Feedback and metal enrichment in cosmological SPH simulations - II. A multiphase model with supernova energy feedback MNRAS 2006 (371) 1125

[13] B. Stern, Y. Tikhomirova, D. Kompaneets, et al., An Off-Line Scan of the BATSE Daily Records and a Large Uniform Sample of Gamma-Ray Bursts ApJ 2001 (563) 80 [astro-ph/ 00094 4]

[14] G. Vedrenne \& J. Atteia, Gamma-Ray Bursts: the brigthest explosions in the Universe, Springer Praxis Books, Jointly published with Praxis Publishing, UK 